Thorax (1969), 24, 750.

\title{
Xiphoid horn in pectus excavatum
}

\author{
E D W A R D F. SK I N NER ${ }^{1}$ \\ From the Department of Surgery, University of Tennessee School of Medicine, \\ Memphis, Tennessee 38103
}

In pectus excavatum there may be a cartilaginous horn on the inner surface of the xiphoid process rubbing on the heart, or the xiphoid process may be tipped inward, rubbing on the heart and causing anginoid pain. Being cartilaginous, these protrusions of the xiphoid cartilage are not visible on preoperative radiographs. Eight per cent of pectus excavatum patients have organic heart disease (Reusch, 1961). Funnel chest may cause anginoid pain (Poppe, 1965) which can be relieved surgically.

A previously unreported abnormality is here recorded. In pectus excavatum there may be a cartilaginous protrusion from the inner surface of the xiphoid cartilage (similar to the horn of a rhinoceros) which rubs on the heart with each beat, or the xiphoid cartilage may be tipped inward so that it rubs on the heart, causing electrocardiographic changes and anginoid pain. Being cartilaginous, these deformities will not show on radiographs.

Two such cases are reported: the first had an actual horn on the inner surface of the xiphoid cartilage, and the second patient had the tip of the xiphoid turned inward so that it also was rubbing on the heart.

\section{OPERATIVE TECHNIQUE}

Neither patient had metal internal or external fixation devices used post-operatively. The operative technique described by Ravitch (1965) was followed, and in addition three stitches of No. 1 cotton were placed beneath the sternum to support it in its new position. These sling stitches run from the pectoral muscles on each side below the sternum.

\section{CASE REPORTS}

CASE 1 Mrs. H. C., a white woman aged 37, gave a history on 4 August 1962 of cough of four weeks' duration and said that when she had colds the cough persisted for a considerable period of time after such colds. Three days before we saw her she fainted. She had momentary 'suppression' sensation in her chest, severe at times, and she felt lifeless. She had had chest pain for a long time on the left running from the front to the back. She had some dyspnoea. Occa-

${ }^{1}$ Requests for reprints to: $20 \mathrm{~S}$. Dudley St., Memphis, Tennessee 38103. U.S.A. sional low-grade fever was present and she also had $\stackrel{\Phi}{3}$ chills. She had gradually lost 10 to $15 \mathrm{lb}$. $(4 \cdot 5$ to $6 \cdot 8$ 음 kg.) in weight and fatigue was marked.

Bronchograms showed minimal bilateral bronchi- $\vec{\bullet}$ ectasis and chest films showed a moderately severe pectus excavatum. Her weight was 7 st. $2 \mathrm{lb}$. $(45 \cdot 3 \cdot$ kg.), height $5 \mathrm{ft} .3$ in. $(1.6 \mathrm{~m}$.). Scattered, medium, coarse, post-tussive râles were present bilaterally, and a moderate pectus excavatum was apparent.

We followed her for three years and the heart became oval-shaped over this period, whereas it was globular on the first examination. Furthermore, it shifted at least 1.5 in. $(38 \mathrm{~mm}$.) more towards the left over this period of time.

On 16 April 1965 repair of the pectus excavatum was carried out in the Baptist Memorial Hospital, as described under 'Operative technique'. There was a peculiar cartilaginous horn on the inner surface of the xiphoid process and this was rubbing on thex heart with each beat. It was difficult for the surgeon to put his finger between the ziphoid process and the heart, it was so tight, and it was actually painful too the surgeon before the sternum was lifted forward $₹$ surgically. This horn was almost half an inch $(120$ $\mathrm{mm}$.) long. The horn was removed but the xiphoid? process itself was not removed, in order to give ao better cosmetic appearance. Her post-operative con valescence was uneventful. We last saw her on $2 \mathrm{~N}$ September 1966. She still had occasional symptoms from her minimal bilateral bronchiectasis and pleurisy. She is leading a normal life.

CASE 2 Mrs. C. W., a 37-year-old white woman was first seen by us on 18 January 1962. She complained of left chest pain anteriorly for several years. 5 Two years previously an intercostal neurectomy had been done in an attempt to relieve the chest pain, but it remained and was aggravated by moving the left arm, and she had had 'bronchial trouble' for? about 18 months. With severe respiratory infections it was necessary for her to sit up to breathe becauseo of dyspnoea. She had lost $13 \mathrm{lb}$. $(5 \cdot 8 \mathrm{~kg}$.) in weight 
during the preceding three years. Her weight was 7 st. ( $44.4 \mathrm{~kg}$.), height $5 \mathrm{ft} .3$ in. $(1.6 \mathrm{~m}$.). She had a marked pectus excavatum and expiratory wheezes bilaterally.

On 1 February 1962 she had a plastic repair of her pectus excavatum in the Baptist Memorial Hospital, as described under 'Operative technique'. At operation the xiphoid process was of normal size but bent inward, causing pressure on the heart with each beat. The xiphoid was fractured but not removed from the sternum, to give a better cosmetic effect. Her convalescence was uneventful and in the next year she gained $30 \mathrm{lb}$. $(13.6 \mathrm{~kg}$.) in weight. She continued to have occasional symptoms from her bronchitis but her cardiac pain was relieved.

\section{DISCUSSION}

A moderate to severe pectus excavatum compresses the heart between the sternum and the spine ; this is apparent on the lateral chest film, and the shape of the heart changes from globular to oval ; the heart also appears to rotate toward the left, and this seems to interfere with its work capacity. Besides the distortion of the heart, the bronchi are sometimes displaced and bronchiectasis may be associated with a pectus excavatum. For the above reasons and because some cases of pectus excavatum are psychologically unacceptable to the patient, most cases of moderate to severe pectus deformities should be repaired surgically. Technically, this is simpler if the patient is 2 years of age or over.

\section{REFERENCES}

Poppe, J. K. (1965). Anginoid pain from funnel chest deformity. Dis. Chest, 47, 667.

Ravitch, M. M. (1965). Technical problems in the operative correction of pectus excavatum. Ann. Surg., 162, 29.

Reusch, C. S. (1961). Hemodynamic studies in pectus excavatum. Circulation, 24, 1143. 